\title{
A 71-year-old woman with recurrent falls and confusion
}

\author{
Mansoor Mehmood MD¹, Omar N Nadhem MD¹, Faisal A Khasawneh MD²
}

\section{CASE PRESENTATION}

A 71-year-old African-American woman presented to the authors' hospital with recurrent falls and confusion. She reported fatigue, loss of appetite and weight loss that had been progressing over several months. In the two days before her presentation, she felt dizzy and the family noticed intermittent confusion. She denied fever, respiratory or urinary symptoms. She reported chronic constipation but no abdominal pain or vomiting. Her medical history was significant for rheumatoid arthritis (RA) and hypertension. The patient's RA had never been treated, despite disease progression over the years; meanwhile, her hypertension was well controlled on hydrochlorothiazide and amlodipine. The patient denied smoking, alcohol abuse or illicit drug use. She also denied any recent medical procedures or dental work.

On examination, she was afebrile, with a temperature of $37.4^{\circ} \mathrm{C}$ and a blood pressure of $96 / 58 \mathrm{mmHg}$. Her heart rate was 92 beats/min and her oxygen saturation was $94 \%$ on room air. She was cachectic with no lymphadenopathy. She was edentulous with no gum line swelling or tenderness. Her skin was intact, with no bruising or rashes. She had clear lung fields, regular heart sounds without murmurs and soft abdomen with no organomegaly. She had obvious chronic joint deformities related to her RA. Her neurological examination was nonfocal.

Laboratory test results revealed a hemoglobin level of $98 \mathrm{~g} / \mathrm{L}$ and white blood cell count of $10.6 \times 10^{9}$ cells/L. Chemistry tests showed a creatinine level of $106.1 \mu \mathrm{mol} / \mathrm{L}$ and albumin level of $19.0 \mathrm{~g} / \mathrm{L}$; alkaline phosphatase, total bilirubin and liver transaminase levels were normal. Computed tomography scans of the brain were negative. Urinalysis and chest $\mathrm{x}$-ray were negative. The anaerobic bottles of the blood culture sets obtained on admission were observed to be positive after $24 \mathrm{~h}$. The Gram stain of the positive bottles is shown in Figure 1.

What is your diagnosis?

\section{DIAGNOSIS}

The Gram stain showed Gram-positive bacilli that were identified to be Clostridium perfringens. The bacterium grew in the automatic blood culture system VersaTrek (TREK Diagnostic Systems, USA) and was identified using the Microscan WalkAway Plus system (Siemens, Germany). Antibiotic susceptibility testing was not performed on the isolated bacteria because the authors' clinical laboratory did not have the capability to perform such testing on anaerobic bacteria. The patient was started on benzathine penicillin $G$ and clindamycin. The patient's history and physical examination was not suspicious for soft tissue, odontogenic or procedural sources of this infection. Based on the above, an underlying abdominal or pelvic pathology, especially a malignancy, was suspected. She underwent computed tomography scan of the abdomen and pelvis with contrast as well as colonoscopy that were negative. She completed a 14-day course of the above-mentioned

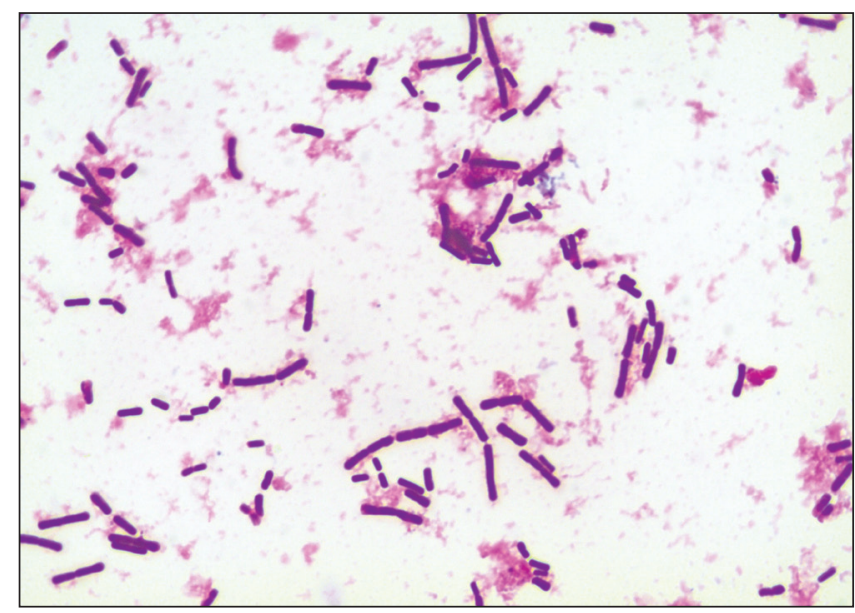

Figure 1) Gram stain of the positive anaerobic blood culture bottle showing Gram-positive boxcar-shaped bacilli

antibiotics with no side effects. The patient's cachexia and chronic constitutional complaints were attributed to undiagnosed depression and she continues to be followed in the outpatient clinic.

\section{DISCUSSION}

Bacteremia due to obligate anaerobes is uncommon. Clostridium species account for $<1 \%$ of positive blood cultures and are second to Bacteroides among isolated anaerobes (1-3). C perfringens is the most commonly isolated Clostridium species in blood cultures. A retrospective populationbased surveillance in Calgary, Alberta, identified 904 cases of anaerobic bacteremia between 2000 and 2008, with an overall population incidence of 8.7 per 100,000 per year (1). The most commonly identified bacteria were Bacteroides fragilis, Clostridium non-perfringens species, Peptostreptococcus species and C perfringens, respectively.

Clostridium species are nonmotile, obligate anaerobic Grampositive bacteria that are capable of forming endospores and are ubiquitous in nitrate-rich environments, such as sewage and soil, as well as in the intestinal tract of humans and animals (2).

$\mathrm{C}$ perfringens has been implicated in causing a wide variety of clinical syndromes ranging from skin and soft tissue infections and gas gangrene, to gastroenteritis and enteritis necroticans (3-6).

After acquiring the bacteria, or its spores, from exogenous or endogenous sources, and in the presence of suitable anaerobic conditions, $\mathrm{C}$ perfringens vegetates and multiplies rapidly, producing a wide variety of toxins that mediate its pathogenicity. Those toxins include

\footnotetext{
${ }^{1}$ Department of Internal Medicine; ${ }^{2}$ Section of Infectious Diseases, Department of Internal Medicine, Texas Tech University Health Sciences Center, Amarillo, Texas, USA

Correspondence: Dr Faisal A Khasawneh, Section of Infectious Diseases, Department of Internal of Medicine, Texas Tech University Health Sciences Center, 1400 South Coulter Street, Amarillo, Texas 79106, USA. Telephone 806-354-5480, fax 806-354-5765, e-mail faisal.khasawneh@ttuhsc.edu
}

OPEN ACCESS This open-access article is distributed under the terms of the Creative Commons Attribution Non-Commercial License (CC BY-NC) (http:// creativecommons.org/licenses/by-nc/4.0/), which permits reuse, distribution and reproduction of the article, provided that the original work is properly cited and the reuse is restricted to noncommercial purposes. For commercial reuse, contact support@pulsus.com 
cytolytic enzymes, enterotoxins, collagenases, proteases and other necrotoxins (7-9).

Clinical correlates determine the exact significance of clostridial bacteremia. It may be a transient bacteremia or, potentially, a pseudobactermia (a contaminant), if the patient had no risk factors or symptoms and signs consistent with an infection (10). More commonly, however, it is a life-threatening event, and the patient is profoundly septic and at high risk for death $(10,11)$. Furthermore, it can be a surrogate of underlying malignancy necessitating further investigation (4).

Reported C perfringens bacteremia risk factors include neutropenia and other immunocompromising disorders, advanced age, hemodialysis and inflammatory bowel diseases (12-16). Poorly attended traumatic wounds and suboptimal surgical techniques can also put the patient at risk for this infection $(5,7,8)$. This bacteremia has rarely complicated routine colonoscopy, evacuating molar pregnancy, choledocholithiasis and strongyloidiasis $(9,12-14)$. It is noteworthy that a substantial number of patients with $\mathrm{C}$ perfringens bacteremia do not have an obvious source (15).

The presentation of $\mathrm{C}$ perfringens bacteremia is nonspecific and depends on disease severity, concomitant infection and organ systems involvement. Nausea, vomiting, abdominal pain, jaundice, loss of appetite and melena are common complaints (5). Other common findings include fever, hypotension and elevated white blood cell counts. A classical picture of $\mathrm{C}$ perfringens septicemia has been described in elderly patients with underlying malignancy, and encompasses abdominal pain and septic shock complicated by severe anemia due to massive intravascular hemolysis caused by a specific clostridial toxin (alpha toxin). Reported mortality rates for the latter condition range from $70 \%$ to $100 \%$ (16).

\section{REFERENCES}

1. Ngo JT, Parkins MD, Gregson DB, et al. Population-based assessment of the incidence, risk factors, and outcomes of anaerobic bloodstream infections. Infection 2013;41:41-8

2. Heikinheimo A, Lindstrom M, Granum PE, Korkeala H. Humans as reservoir for enterotoxin gene - carrying Clostridium perfringens type A. Emerg Infect Dis 2006;12:1724-9.

3. Leal J, Gregson DB, Ross T, Church DL, Laupland KB. Epidemiology of Clostridium species bacteremia in Calgary, Canada, 2000-2006. J Infect 2008;57:198-203.

4. Rechner PM, Agger WA, Mruz K, Cogbill TH. Clinical features of clostridial bacteremia: A review from a rural area. Clin Infect Dis 2001;33:349-53.

5. Fujita H, Nishimura S, Kurosawa S, Akiya I, Nakamura-Uchiyama F, Ohnishi K. Clinical and epidemiological features of Clostridium perfringens bacteremia: A review of 18 cases over 8 year-period in a tertiary care center in metropolitan Tokyo area in Japan. Intern Med 2010;49:2433-7.

6. Juntermanns B, Radunz S, Heuer M, et al. Fulminant septic shock due to Clostridium perfringens skin and soft tissue infection eight years after liver transplantation. Ann Transplant 2011;16:143-6.

7. Chen YM, Lee HC, Chang CM, Chuang YC, Ko WC. Clostridium bacteremia: Emphasis on the poor prognosis in cirrhotic patients. J Microbiol Immunol Infect 2001;34:113-8.

8. Tabarelli W, Bonatti H, Cejna M, Hartmann G, Stelzmueller I, Wenzl E. Clostridium perfringens liver abscess after pancreatic resection. Surg Infect (Larchmt) 2009;10:159-62.
Successful management of this infection requires prompt recognition and initiation of appropriate antimicrobial therapy, among other support measures, in addition to surgical intervention and debridement when necessary. For isolated $\mathrm{C}$ perfringens bacteremia, penicillin is considered to be the drug of choice, either alone or in combination with clindamycin, although emergence of resistance against the latter has been reported (3). Ngo et al (1) reported the susceptibility of $71 \mathrm{C}$ perfringens blood isolates to penicillin, metronidazole and clindamycin (1). None of the above isolates were resistant to penicillin or metronidazole, although $11(15.5 \%)$ were resistant to clindamycin. Other commonly used effective antibiotics include ampicillin/sulbactam and piperacillin/ tazobactam (4). Exchange transfusion and hyperbaric oxygen therapy may be of help in bacteremia cases complicating skin or soft tissue infections, especially if shock and hemolysis are evident (17). The latter intervention disrupts the bacterial anaerobic environment and, hence, facilitates eradication of the microbe in small abscesses and ischemic infected tissues.

Poor prognostic factors include old age, elevated serum fibrinogen levels, septic shock, nosocomial acquisition of infection, hemolysis, polymicrobial bacteremia and insufficient antimicrobial coverage (11). Despite appropriate therapy, the overall mortality of this rare bacteremia continues to be high $(3,4,10,11)$. In the abovementioned retrospective surveillance, the overall case fatality rate of anaerobic bacteremia was $20 \%$ (1). The case fatality rate was higher in C perfringens bacteremia (29.7\%) than in B fragilis cases (16.6\%). This higher mortality was attributed to the multiple cytotoxic virulence factors produced by $\mathrm{C}$ perfringens.

DISCLOSURES: The authors have no conflicts of interest to declare.

9. Adams BN, Lekovic JP, Robinson S. Clostridium perfringens sepsis following a molar pregnancy. Am J Obstet Gynecol 2014;210:e13-4.

10. Yang CC, Hsu PC, Chang HJ, Cheng CW, Lee MH. Clinical significance and outcomes of Clostridium perfringens bacteremia a 10-year experience at a tertiary care hospital. Int J Infect Dis 2013;17:e955-60.

11. Shah M, Bishburg E, Baran DA, Chan T. Epidemiology and outcomes of clostridial bacteremia at a tertiary-care institution. ScientificWorldJournal 2009;9:144-8.

12. Shea YF, Chau KM, Hung IF, Chu LW. Strongyloidiasis in a nonagenarian who previously worked in conservancy services. Hong Kong Med J 2013;19:74-6.

13. Kunz AN, Riera D, Hickey P. Case of Clostridium perfringens bacteremia after routine colonoscopy and polypectomy. Anaerobe 2009;15:195-6.

14. Atia A, Raiyani T, Patel P, Patton R, Young M. Clostridium perfringens bacteremia caused by choledocholithiasis in the absence of gallbladder stones. World J Gastroenterol 2012;18:5632-4.

15. Nelson RM, Wilson RF, Osmer RL. Clostridium perfringens bacteremia. Opportunist or killer? Am Surg 1985;51:301-3.

16. Pirrotta MT, Bucalossi A, Forconi F, et al. Massive intravascular hemolysis: A fatal complication of Clostridium perfringens septicemia in a patient with acute lymphoblastic leukemia. Leuk Lymphoma 2005;46:793.

17. Rajendran G, Bothma P, Brodbeck A. Intravascular haemolysis and septicaemia due to Clostridium perfringens liver abscess. Anaesth Intensive Care 2010;38:942-5. 


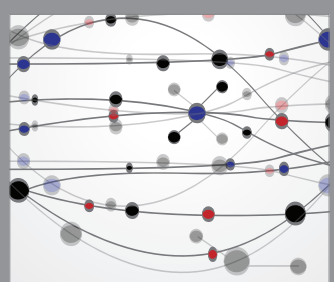

The Scientific World Journal
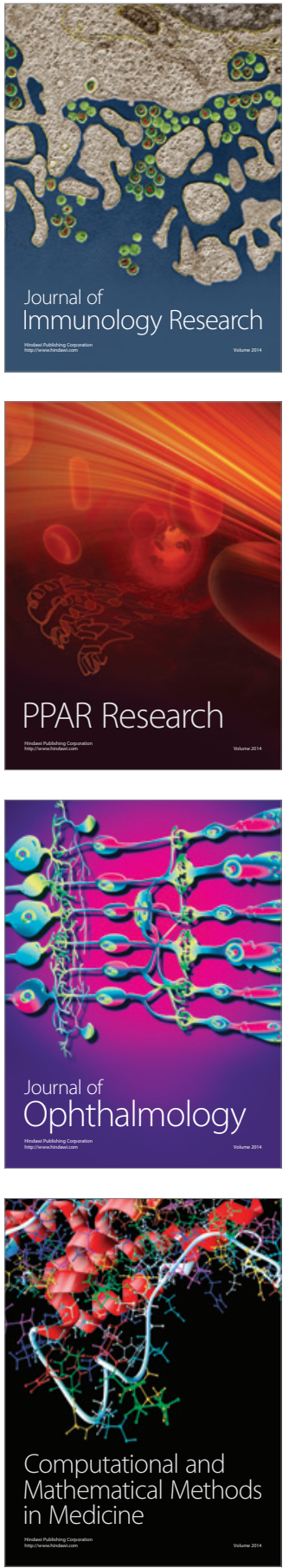

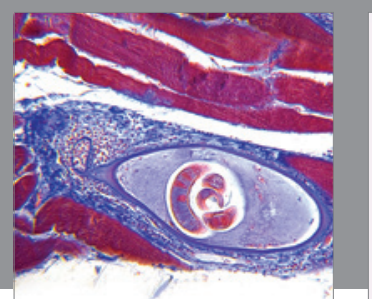

Gastroenterology Research and Practice

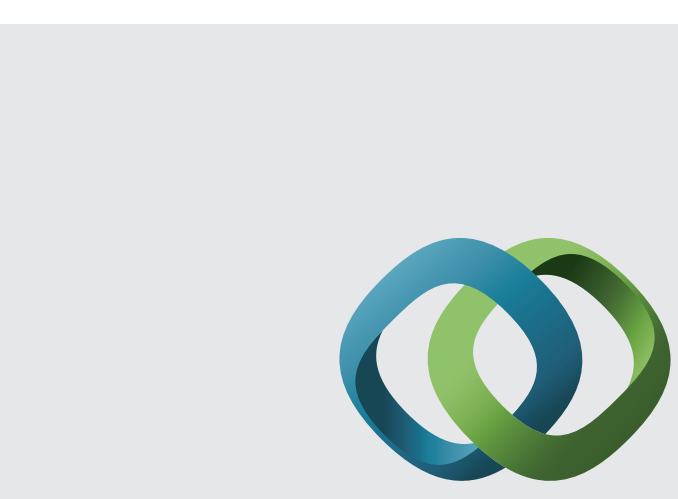

\section{Hindawi}

Submit your manuscripts at

http://www.hindawi.com
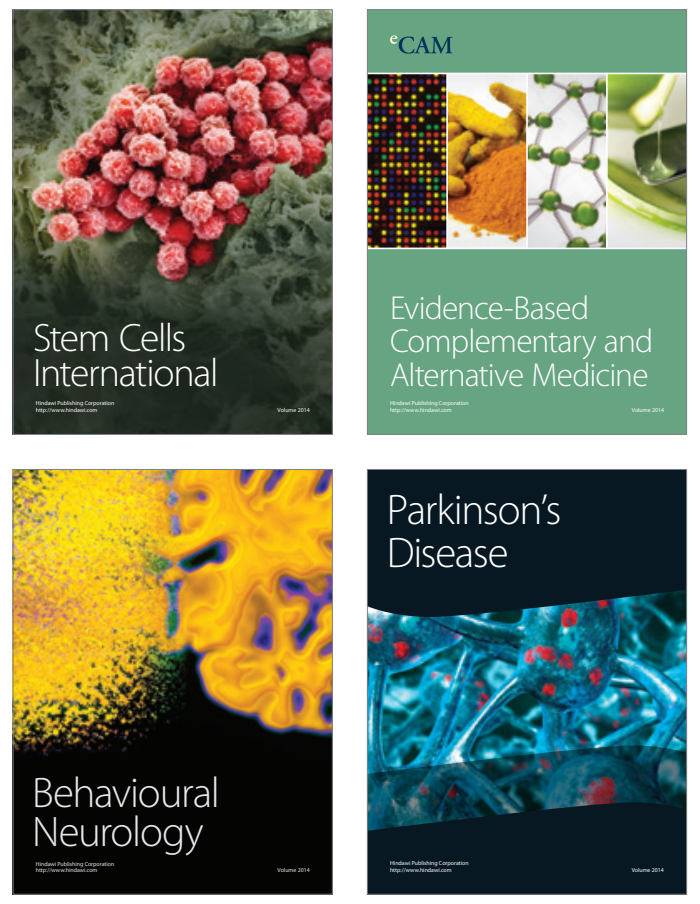
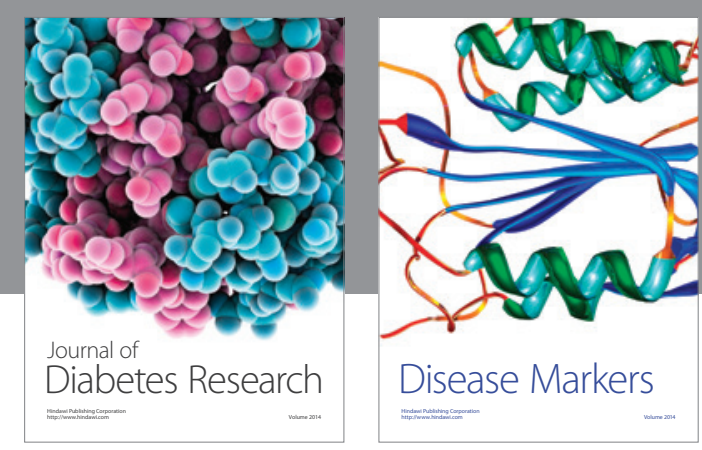

Disease Markers
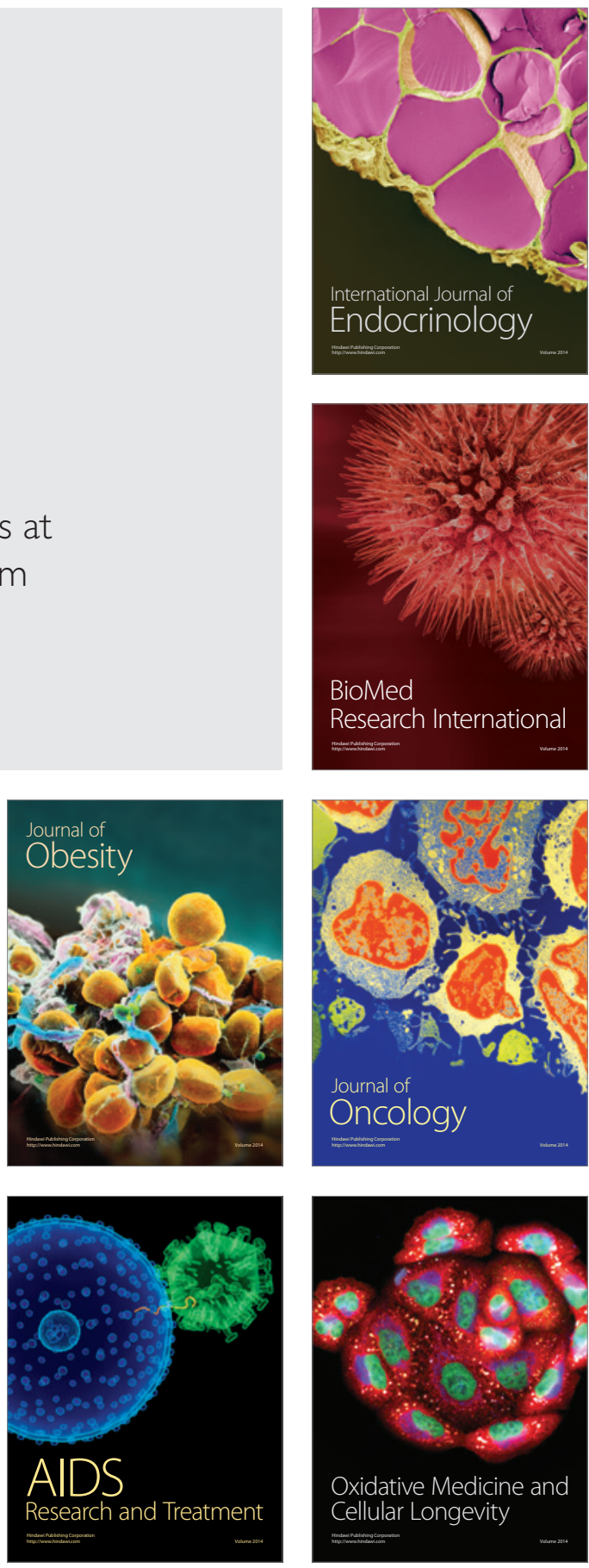